\title{
Dynamic Routing and Wavelength Assignment in Multifiber WDM Networks with Wavelength Conversion Capability
}

\author{
Kouji Hirata ${ }^{1} \&$ Dewiani $^{2}$ \\ ${ }^{1}$ Faculty of Engineering, TokyoUniversity of Science, Tokyo, Japan \\ ${ }^{2}$ Graduate School of Science and Engineering, Ehime University, Matsuyama, Japan \\ Correspondence: Kouji Hirata, Faculty of Engineering, Tokyo University of Science, Tokyo 102-0073, Japan. Tel: \\ 81-3-3260-4271. E-mail: hirata@ee.kagu.tus.ac.jp
}

\author{
Received: September 3, 2012 Accepted: September 21, $2012 \quad$ Online Published: October 7, 2012 \\ doi:10.5539/nct.v1n2p36 \\ URL: http://dx.doi.org/10.5539/nct.v1n2p36
}

\begin{abstract}
This paper proposes a dynamic routing and wavelength assignment (RWA) scheme in multifiber WDM networks with wavelength conversion capability. In multifiber WDM networks, each link consists of multiple fibers. Thus, multiple lightpaths with the same wavelength can be established in the same link as long as they use different fibers. In WDM networks with wavelength conversion, the network nodes have wavelength conversion capability which can convert one wavelength to another. In order to efficiently utilize multifiber and wavelength conversion capability, an appropriate RWA scheme is necessary. The proposed scheme provides RWA for multifiber WDM networks with sparse and full wavelength conversion capability. In the proposed scheme, a route and wavelengths are selected for each lightpath based on wavelength availability and location of nodes with wavelength conversion capability. Through simulation experiments, we show that the proposed scheme reduces the blocking probability of lightpath establishment efficiently.
\end{abstract}

Keywords: multifiber WDM optical networks, routing and wavelength assignment, dynamic lightpath establishment, wavelength conversion

\section{Introduction}

In recent years, WDM optical networks have been expected as a platform for next-generation networks to support the explosive increase in traffic volume on the Internet. The WDM technology increases the capacity of a fiber optic link by simultaneously transmitting multiple signals along lightpaths with different wavelengths over a single fiber (Karasan \& Banerjee, 1998). Furthermore, multifiber environments enhance the performance of the WDM networks such as the blocking probability of lightpath establishment (Baroni et al., 1999; Li \& Somani, 2000; Nomikos et al., 2006; Rahbar, 2010). In multifiber WDM networks, each link consists of multiple fibers, and thus multiple lightpaths with a common wavelength can be established in the same link as long as they use different fibers. Moreover, wavelength conversion techniques which convert one wavelength to another at intermediate nodes can also enhance the performance of WDM networks (Chu et al., 2003; Ramamurthy \& Mukherjee, 1998; Subramaniam et al., 1996).

In this paper, we focus on full and sparse wavelength conversion. A WDM network is referred to as a network with full wavelength conversion if all nodes have wavelength conversion capability. On the other hand, in a WDM network with sparse wavelength conversion, only a subset of the network nodes has wavelength conversion capability. Furthermore, we can categorize wavelength conversion capability into full-range and limited-range wavelength conversion. The full-range wavelength conversion means that a given wavelength can be converted to any wavelength. In contrast, if a given wavelength can be converted to a limited number of wavelengths at anode with wavelength conversion capability, the wavelength conversion technology is referred to as limited-range wavelength conversion. In this paper, we assume that nodes with wavelength conversion capability can convert a wavelength to any wavelength (i.e., full-range wavelength conversion). Under this assumption, we aim to enhance the performance of multifiber WDM networks with full and sparse wavelength conversion.

In order to efficiently utilize these multifiber and wavelength conversion environments, an appropriate routing and wavelength assignment (RWA) scheme is necessary (Mokhatar \& Azizoglu, 1998; Zang et al., 2000). 
Although RWA is categorized to static and dynamic, we focus on dynamic RWA in which lightpaths are dynamically established as lightpath-setup requests arrive (Zang et al., 2001). The performance metric in networks using dynamic RWA is typically the blocking probability of lightpath establishment. A lightpath must use a common wavelength in all links along a route if there are no nodes with wavelength conversion capability along the route (i.e., wavelength continuity constraint). Thus we need an appropriate RWA scheme which considers location of nodes with wavelength conversion capability in order to reduce the blocking probability of lightpath establishment.

In the past, several dynamic RWA schemes have been proposed for multifiber WDM networks (Coiro et al., 2011a; Coiro et al., 2011b; Dewiani et al., 2011; Kim et al., 2006; Liu, 2009; Xu et al., 2000). The authors in (Coiro et al., 2011a; Coiro et al., 2011b) provided power-aware dynamic RWA which considers not only blocking performance but also power consumption and energy efficiency in multifiber WDM networks. In Dewiani et al. (2011), the authors proposed a dynamic RWA scheme which selects a combination of a route and a wavelength based on wavelength availability collected by backward reservation signaling. In Kim et al. (2006), the authors proposed three dynamic RWA schemes named MCR, LSNLR, and $F(w, l)$. These schemes first select a route among the pre-determined routes and then they select a wavelength based on wavelength availability. The author in Liu (2009) proposed an algorithm named ICJ which avoids the generation of bottleneck links by appropriately assigning wavelengths. In Xu et al. (2000), the authors proposed two RWA schemes named PACK and SPREAD. They select a combination of a route and a wavelength based on some metric associated with each combination. Although these RWA schemes enhance the performance of multifiber WDM networks, they do not consider wavelength conversion capability. Therefore, they cannot be directly applied to multifiber WDM networks with wavelength conversion.

This paper proposes a dynamic RWA scheme for multifiber WDM networks with wavelength conversion. In the proposed scheme, a route and wavelengths are selected for each lightpath based on wavelength availability and location of nodes with wavelength conversion capability. Specifically, the proposed scheme divides each route into segments between nodes with wavelength conversion. Then, the proposed scheme selects a route based on wavelength availability in segments and assigns a least-used wavelength to each segment on the selected route. By doing so, the proposed scheme aims to avoid the depletion of a specific wavelength in each link and the generation of bottleneck links. As a result, the proposed scheme is expected to reduce the blocking probability of lightpath establishment effectively in multifiber WDM networks with wavelength conversion.

The rest of this paper is organized as follows. In Section 2, we explain multifiber WDM networks and wavelength conversion. Section 3 explains our proposed scheme. In Section 4, the performance of the proposed scheme is discussed with the results of simulation experiments. Finally, we conclude the paper in Section 5.

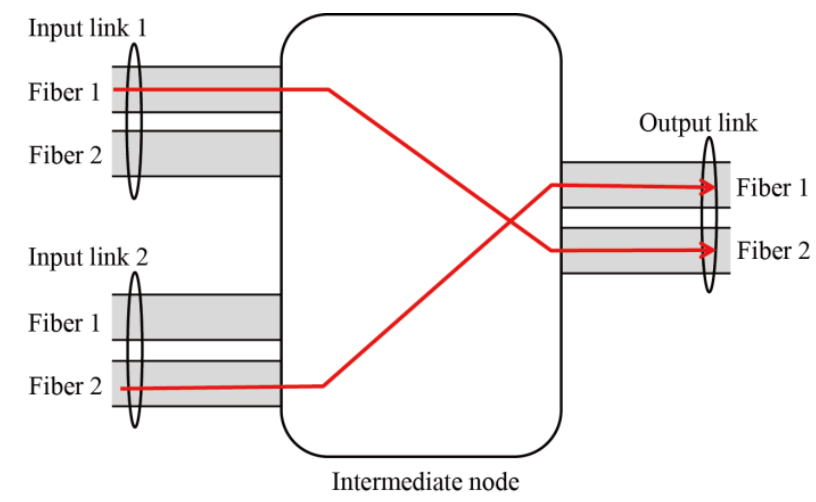

Figure 1. Intermediate node in multifiber WDM networks

\section{Multifiber WDM Networks with Wavelength Conversion}

In multifiber WDM networks, multiple lightpaths can be established with the same wavelength in the same link as shown in Figure 1, where each link has two fibers. In this figure, we assume that a lightpath with a given wavelength from fiber 1 in input link 1 to fiber 2 in the output link has already been established at the intermediate node. If the number of fibers in the output link is 1, i.e., single fiber link, a new lightpath for the output link cannot be established with the same wavelength. On the other hand, in multifiber networks, a new lightpath with the same wavelength can be established through a different fiber of the output link as long as the 
wavelength is available in the fiber as shown in the figure. Thus multifiber environments improve the blocking performance of WDM networks.

The wavelength conversion technology further improves the blocking performance of WDM networks. In sparse wavelength conversion networks, a subset of the network nodes has full-range wavelength conversion capability. On the other hand, in full wavelength conversion networks, all network nodes have full-range wavelength conversion capability. Specifically, in the network nodes, a given wavelength can be converted to any wavelength. We show an example with Figure 2, where each link has two fibers. In this figure, we assume that fibers 1 and 2 in the output link have already been used by lightpaths with wavelength $w_{1}$. In this case, a new lightpath with the same wavelength cannot be established in the output link. Thus, the new lightpath establishment with wavelength $w_{1}$ is blocked if the node does not have wavelength conversion capability. On the other hand, if the node has wavelength conversion capability, the new lightpath can be established by converting an input wavelength (i.e., $w_{1}$ in the figure) to an available wavelength (i.e., $w_{2}$ in the figure).

There are some wavelength converter architectures such as a dedicated wavelength converter, a share-per-node wavelength converter, and a share-per-link wavelength converter (Ramamurthy \& Mukherjee, 1998). In the dedicated wavelength converter architecture, each signal in each fiber has a dedicated wavelength converter. Specifically, a wavelength of incoming optical signal can be always converted to any wavelength as long as the wavelength is available at the output fiber. In the share-per node wavelength converter architecture and the share-per-link wavelength converter architecture, wavelength converters are shared with signals in a node and an output fiber link, respectively. In this paper, we use the dedicated wavelength converter architecture for nodes with wavelength conversion capability.

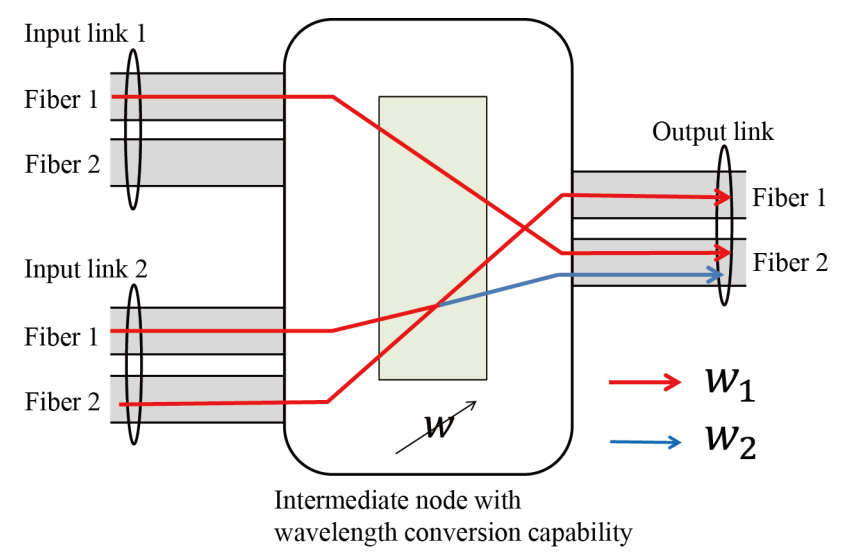

Figure 2. Intermediate node with wavelength conversion capability

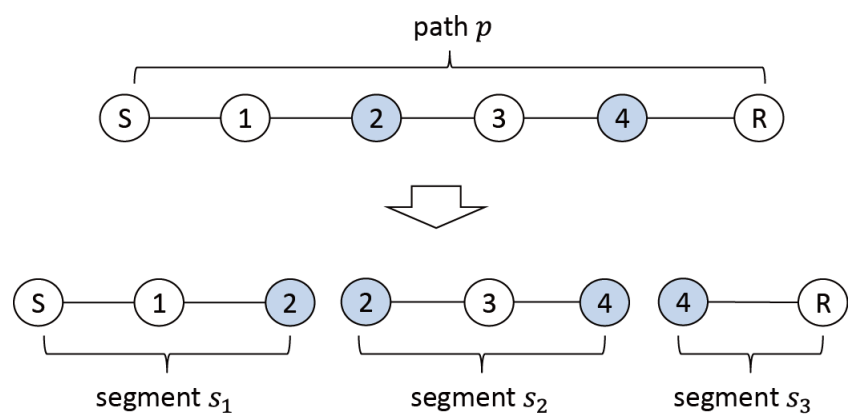

Figure 3. Segments (nodes 2 and 4 have wavelength conversion capability)

\section{Proposed Scheme}

\subsection{Overview}

The proposed scheme provides an RWA approach for multifiber WDM networks with wavelength conversion. In the proposed scheme, a route between a sender node and a receiver node is selected from a set of pre-defined routes which are link-disjoint paths, i.e., paths which do not share a link, and a wavelength is selected along the selected route. The proposed scheme considers location of nodes with wavelength conversion capability in route 
and wavelength selection. In particular, the proposed scheme divides each path to segments between nodes with wavelength conversion capability as shown in Figure 3. Then the proposed scheme selects a route among pre-defined paths and assigns wavelengths to segments along the selected route in such a way as to avoid the generation of bottleneck links and the depletion of a specific wavelength.

To do so, the proposed scheme selects a route based on wavelength availability in each segment. A bottleneck link is generated when traffic concentrates in a certain link and all wavelengths in the link are in simultaneous use. In this case, further lightpaths cannot be established in the link. Therefore, we expect to reduce the blocking probability by avoiding the generation of bottleneck links. In order to distribute loads and avoid the generation of bottleneck links, the proposed scheme preferentially selects a route which has segments with many available wavelengths.

Furthermore, it is not preferable that a specific wavelength is used in all fibers on a link. A lightpath must use a common wavelength along a segment due to wavelength continuity constraint. Therefore, a wavelength cannot be used in cases where the wavelength is already used in all fibers on a certain link of a segment even if the wavelength is available in other links of the same segment. Thus, in order to avoid this situation, the proposed scheme selects a route based on usage of each wavelength in each link and preferentially assigns a least-used wavelength to each segment on the selected route.

\subsection{The Procedure of the Proposed Scheme}

In what follows, we explain the detail of the proposed scheme.

\subsubsection{Construction of Pre-defined Paths}

The proposed scheme selects a route from a set $P$ of pre-defined link-disjoint paths. In order to construct those pre-defined paths, the proposed scheme adopts the following simple algorithm (Dewiani et al., 2011) for each pair of a sender node and a receiver node. Let $G=(V, E)$ denote a directed graph, where $V$ and $E$ denote sets of nodes and links, respectively. At first, the proposed scheme finds the shortest path from a sender node to a receiver node on $G$, using Dijkstra's algorithm, and adopt the path as a link-disjoint path. Then the links along the path are removed from $G$. The proposed scheme finds the new shortest path on the resulting graph and the path is adopted as a new link-disjoint path. The procedure is repeated as long as there are routes from the sender node to the receiver node.

\subsubsection{Route and Wavelength Selection}

The proposed scheme divides each path $p \in P$ between a sender node and a receiver node to segments $s_{i}(i=1$, $\left.2, \ldots, N_{p}+1\right)$ between nodes with wavelength conversion, where $N_{p}$ denotes the number of intermediate nodes with wavelength conversion capability along the path $p$. Note that the starting point of $s_{1}$ is the sender node and the ending point of $s_{N p+1}$ is the receiver node. We define $\operatorname{cost} C_{p}$ of path $p \in P$ as

$$
\mathrm{C}_{\mathrm{p}}=\sum_{s \in p}\left\{\frac{\min _{w \in A_{s}} c_{w, s}}{\left|A_{s}\right|}\right\},
$$

where $A_{s}$ denotes the set of available wavelengths in segment $s$, and $c_{w, s}$ denotes the cost of wavelength $w$ in segment $s$. We define $c_{w, s}$ as follows:

$$
c_{w, s}=\left\{\begin{array}{c}
\sum_{l \in E_{s}}\left\{n_{w, l} \times \frac{\sum_{f \in F_{l}} u_{l, f}}{\sum_{f \in F_{l}} W_{l, f}}\right\}, \text { if } n_{w, l}<\left|F_{l}\right| \\
\infty, \text { otherwise }
\end{array}\right.
$$

where $E_{s}$ denotes the set of links in segment $s, n_{w, l}$ denotes the number of link $l$ 's fibers in which wavelength $w$ is already used, $F_{l}$ denotes the set of fibers in link $l, u_{l, f}$ denotes the number of used wavelengths in fiber $f$ on link $l$, and $W_{l, f}$ denotes the number of wavelengths supported by fiber $f$ on link $l$. Note that these information along routes is collected by some schemes (e.g., Dewiani et al., 2011).

The proposed scheme selects a path with the smallest cost $C_{p}$ as a route for a new lightpath. If $C_{p}$ is infinity for all paths, the new lightpath establishment is blocked. Note that if there are two or more paths with the minimum cost, the proposed scheme selects a path with shorter hops. If the numbers of hops among them are the same, the proposed scheme selects one randomly. Also, the proposed scheme assigns a wavelength with the smallest cost to each segment $s \in p$ along the selected path $p$. Specifically, the proposed scheme uses wavelengths selected in 
(1), i.e., wavelength $w$ with the smallest $\operatorname{cost} c_{w, s}$ in each segment $s \in p$. If there are multiple candidates, the proposed scheme selects one randomly. We summarize the procedure of the proposed scheme in Figure 4.

Step 1. For each sender-receiver pair, a set $P$ of pre-defined link-disjoint paths is constructed from a directed graph $G=(V, E)$.

Step 2.Each path $p \in P$ between a sender node and a receiver node is divided into segments $s_{i}$ between nodes with wavelength conversion.

Step 3. Whenever a lightpath-setup request arrives, a route and wavelengths are selected as follows.

Step 3-1. For each path $p \in P$ between the sender and the receiver nodes of the lightpath, the $\operatorname{cost} C_{p}$ is calculated with (1) and (2), and the path with the smallest cost is selected as a candidate route.

Step 3-2. The wavelength $w$ with the smallest $\operatorname{cost} c_{w, s}$, which is calculated with (2), is selected in each segments along the selected route.

Step 3-3. The lightpath is established with the selected wavelengths along the selected route.

Figure 4. The procedure of the proposed scheme

As it can be seen from (1) and (2), the cost increases with the sum $\sum_{f \in F} u_{l, f}$ of numbers of wavelengths used in link $l$. Also, the cost decreases with the increase in the number $\left|A_{s}\right|$ of available wavelengths in segment $s$. Thus, the proposed scheme tends to select a route which has segments with many available wavelengths as mentioned in Section 3.1. Moreover, the cost increases with the number $n_{w, l}$ of link l's fibers in which wavelength $w$ is used. Therefore, we affirm that the proposed scheme selects a route based on usage of each wavelength in each link.

\subsection{An Example of the Proposed Scheme}

We show an example of the proposed scheme in Figure 5, where $U_{i}(i=1,2,3)$ in each link denotes the set of already used wavelengths in fiber $i$. We assume that each link consists of three fibers and each fiber supports three wavelengths $w_{1}, w_{2}$, and $w_{3}$. In this figure, there are two pre-defined paths $p$ and $p^{\prime}$ between the sender node and the receiver node. Path $p$ is divided into segments $s_{1}$ and $s_{2}$ by the node with wavelength conversion capability (denoted by node with WC). Segments $s_{1}$ includes links $l_{1}$ and $l_{2}$, and segment $s_{2}$ includes link $l_{3}$. Also, path $p^{\prime}$ is divided into segmentss ${ }_{1}^{\prime}$ and $s_{2}{ }_{2}$. Segments $s_{1}^{\prime}$ and $s_{2}^{\prime}$ include links $l_{4}$ and $l_{5}$, respectively.

We first calculate the cost $C_{w 1, s 1}$ of wavelength $w_{1}$ for segment $s_{1}$ from (2). Segment $s_{1}$ consists of links $l_{1}$ and $l_{2}$ (i.e., $l_{1}, l_{2} \in E_{s 1}$ ). As we can see from Figure $4, n_{w 1, l 1}=0, \sum_{f \in F l 1} u_{l 1, f}=2$, and $\sum_{f \in F l 1} W_{l 1, f}=9$. Similarly, $n_{w 1, l 2}=1$, $\sum_{f \in F l 2} u_{l 2, f}=3$, and $\sum_{f \in F l 2} W_{l 2, f}=9$. Thus, the cost $C_{w 1, s 1}$ of wavelength $w_{1}$ in segment $s_{1}$ is $0 \times 2 / 9+1 \times 3 / 9=1 / 3$. Also, we can calculate cost $c_{w, s}$ of each wavelength $w$ in each segment $p$ as shown in Table 1 . Note that $C_{w 1, s 2}$ and $C_{w 3, s^{\prime} 2}$ are $\infty$ because wavelengths $w_{1}$ and $w_{3}$ cannot be used in links $l_{3}$ and $l_{5}$, respectively.

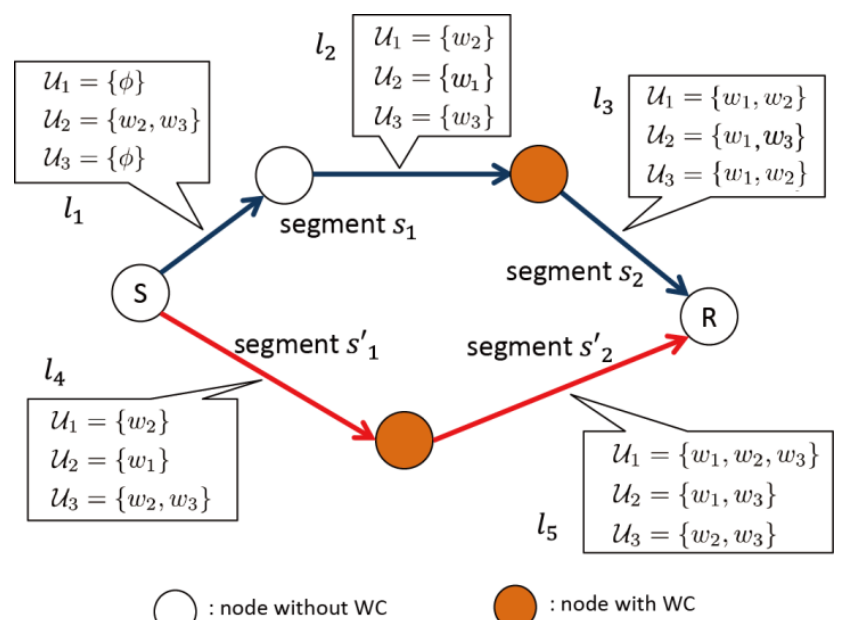

Figure 5. An example of the proposed scheme

In segment $s_{1}$, wavelength $w_{1}$ is selected because $C_{w 1, s 1}$ is the smallest in segment $s_{1}$. Furthermore, in segment $s_{2}$, wavelength $w_{3}$ is selected. Since wavelength $w_{1}$ cannot be used in link $l_{3}$ of segment $s_{2}$ (i.e., $\left.A_{s 2}=\left\{w_{2}, w_{3}\right\}\right),\left|A_{s 1}\right|$ and $\left|A_{s 2}\right|$ are 3 and 2, respectively. As a result, $C_{p}$ is (1/3)/3+(2/3)/2=4/9 (see (1)). Similarly, we can calculate 
the cost of $C_{p^{\prime}}$. In this example, $C_{p^{\prime}}$ is $(4 / 9) / 3+(14 / 9) / 2=25 / 27$ and $C_{p}$ is smaller than $C_{p^{\prime}}$. Thus, the proposed scheme selects path $p$ and assigns wavelengths $w_{1}$ and $w_{3}$ to segments $s_{1}$ and $s_{2}$, respectively.

Table 1. Cost of the example

\begin{tabular}{lcclcc}
\hline Segment & Cost & Value & Segment & Cost & Value \\
\hline \multirow{3}{*}{$s_{1}$} & $C_{w_{1}, s_{1}}$ & $0 \times 2 / 9+1 \times 3 / 9=1 / 3$ & & $C_{w_{1}, s_{1}^{\prime}}$ & $1 \times 4 / 9=4 / 9$ \\
& $C_{w_{2}, s_{1}}$ & $1 \times 2 / 9+1 \times 3 / 9=5 / 9$ & $s^{\prime}{ }_{1}$ & $C_{w_{2}, s_{1}^{\prime}}$ & $2 \times 4 / 9=8 / 9$ \\
& $C_{w_{3}, s_{1}}$ & $1 \times 2 / 9+1 \times 3 / 9=5 / 9$ & & $C_{w_{3}, s_{1}^{\prime}}$ & $1 \times 4 / 9=4 / 9$ \\
\hline \multirow{3}{*}{$s_{2}$} & $C_{w_{1}, s_{2}}$ & $\infty$ & & $C_{w_{1}, s_{2}^{\prime}}$ & $2 \times 7 / 9=14 / 9$ \\
& $C_{w_{2}, s_{2}}$ & $2 \times 6 / 9=4 / 3$ & $s^{\prime}{ }_{2}$ & $C_{w_{2}, s_{2}^{\prime}}$ & $2 \times 7 / 9=14 / 9$ \\
& $C_{w_{3}, s_{2}}$ & $1 \times 6 / 9=2 / 3$ & & $C_{w_{3}, s_{2}^{\prime}}$ & $\infty$ \\
\hline
\end{tabular}

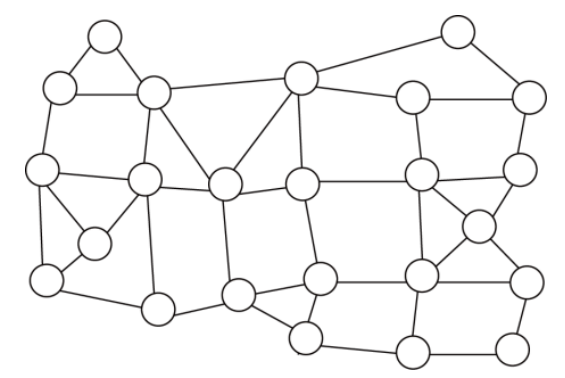

Figure 6. Network model

\section{Performance Evaluation}

\subsection{Model}

To evaluate the performance of the proposed scheme, we conduct simulation experiments with the network topology shown in Figure 6. It consists of 24 nodes and 43 bidirectional links, and $N$ nodes have wavelength conversion capability. If $N$ is 24 , the network has full wavelength conversion capability. If $N$ is 0 , there are no nodes with wavelength conversion capability. For simplicity, we assume that the propagation delay of each link is equal to 0.1 [msec], and processing time of signaling at each node is 0.01 [msec]. Holding time of each lightpath follows an exponential distribution with mean $L=1000$ [sec]. At each node, lightpath-setup requests are generated according to a Poisson process with rate $\lambda$. The destination of each request is independently chosen equally likely among all possible nodes. As a signaling protocol to establish lightpaths, we use wavelength routing with backward reservation (Yuan et al., 1999). We define $\rho$ as the offered load per wavelength on a fiber:

$$
\rho=\frac{\lambda L}{F W}
$$

where $W$ denotes the number of wavelengths supported by each fiber and $F$ denotes the number of fibers in each link. Under the above assumption, we conduct simulation experiments with the self-produced $\mathrm{C}++$ network simulator. As a computer for simulation experiments, we use DELL PRECISION T7500 with Intel Xeon 2.80 $\mathrm{GHz}$ CPU and 12 Gbyte RAM. We collect 30 independent samples from simulation experiments, and 95\% confidence intervals are shown (even though most of them are invisible).

For the sake of comparison, we use the following two RWA schemes. In the first RWA scheme, a path with the shortest hops is preferentially selected. Then the selected path is divided into segments between nodes with wavelength conversion capability, similar to the proposed scheme. This scheme assigns a randomly selected wavelength to each segment. We refer to this scheme as SR (shortest path routing and random wavelength assignment), hereafter.

The second RWA scheme is WLCR-FF (Chu et al., 2003). This scheme is used in single fiber WDM networks with sparse or full wavelength conversion. In this scheme, a path which has many available wavelengths is preferentially selected and a wavelength for each segment in the selected path is assigned in a first-fit manner. 


\subsection{Simulation Results}

\subsubsection{Sparse Wavelength Conversion Network}

First, we examine the performance of the proposed scheme under sparse wavelength conversion environments. To do so, we randomly select 8 nodes as nodes with wavelength conversion capability (i.e., $N=8$ ). Figure 7 shows the blocking probability of lightpath establishment as a function of the offered load $\rho$, where $W=4$ and $F=4$. Note that the blocking probability of lightpath establishment is defined as

$$
\text { blocking probability of lightpath establishment }=\frac{\text { the number of blocked lightpath-setup requests }}{\text { the total number of lightpath-setup requests }}
$$

As it can be seen from Figure 7, the blocking probability of SR is high because it does not consider wavelength availability and frequently generates bottleneck links. On the other hand, WLCR-FF and the proposed scheme reduce the blocking probability of lightpath establishment because they effectively utilize wavelength resources. We also observe that the proposed scheme reduces the blocking probability more efficiently than WLCR-FF. This is because WLCR-FF does not consider multifiber environments. On the other hand, the proposed scheme selects routes and wavelengths, considering wavelength availability in fibers. Thus the proposed scheme can utilize wavelength resources more efficiently than WLCR-FF in multifiber WDM networks. As a result, the proposed scheme exhibits an excellent performance.

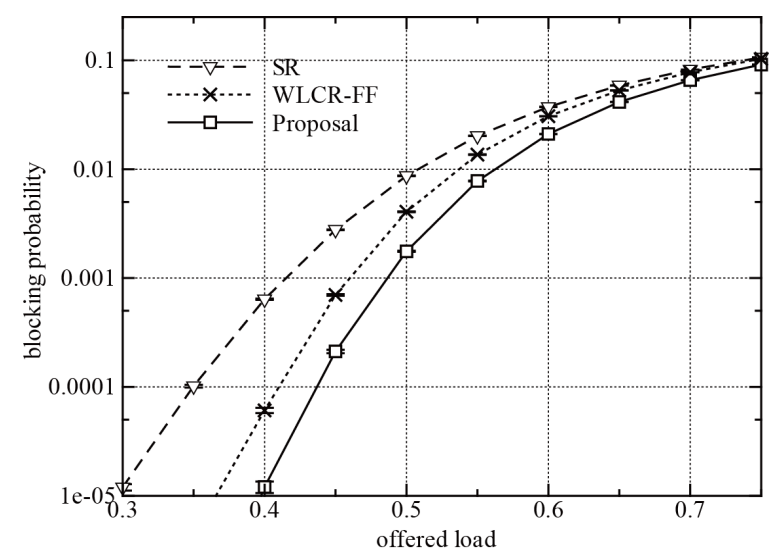

Figure 7. Blocking probability $(\mathrm{W}=4, \mathrm{~F}=4)$ (sparse wavelength conversion)

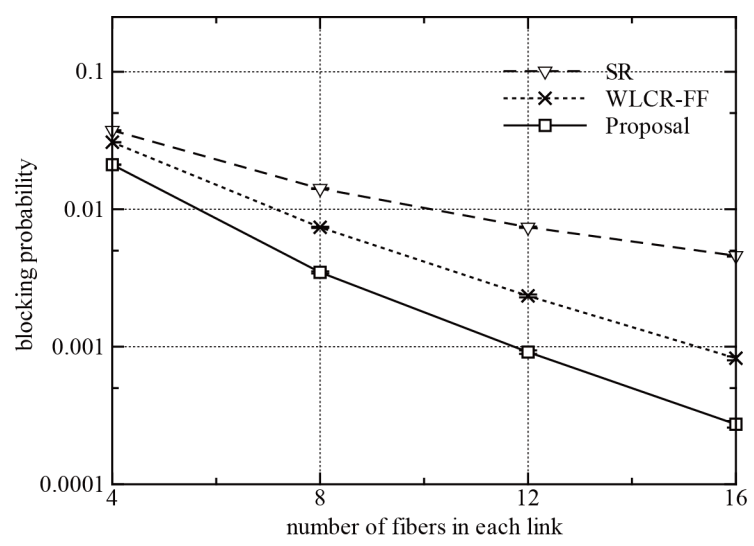

Figure 9. Blocking probability $(\mathrm{W}=4, \rho=0.6)$ (sparse wavelength conversion)

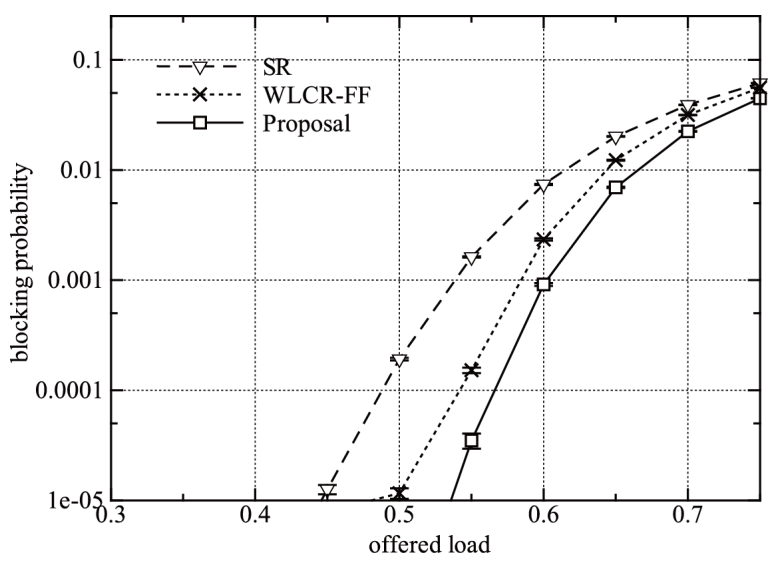

Figure 8. Blocking probability $(\mathrm{W}=4, \mathrm{~F}=12)$ (sparse wavelength conversion)

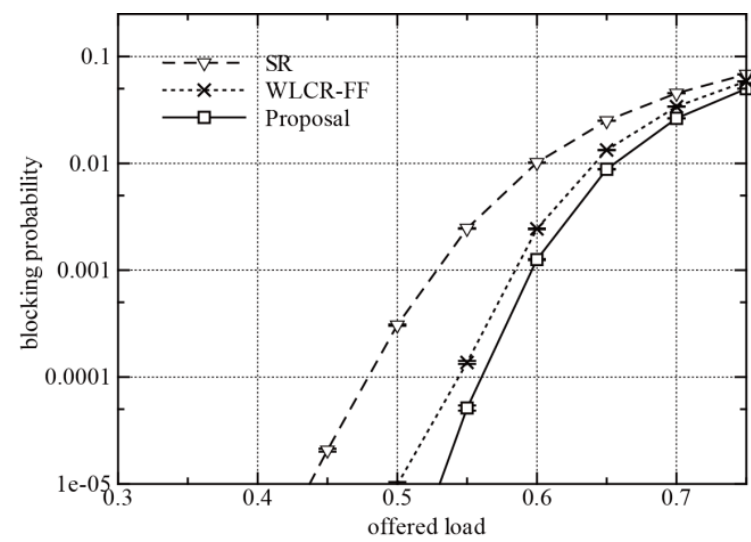

Figure 10. Blocking probability $(\mathrm{W}=12, \mathrm{~F}=4)$ (sparse wavelength conversion) 


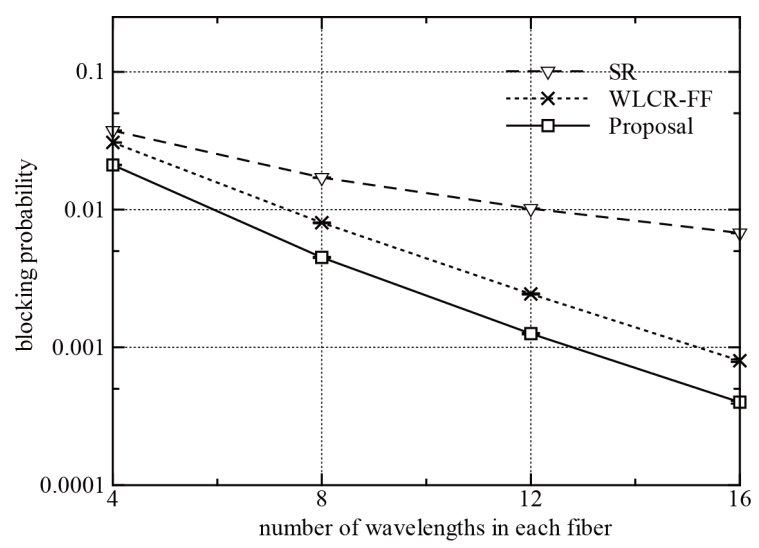

Figure 11. Blocking probability $(F=4, \rho=0.6)$ (sparse wavelength conversion)

We then demonstrate the robustness of the superior performance of the proposed scheme against system parameter values such as the number $W$ of wavelengths supported by each fiber and the number $F$ of fibers in each link.

First, we examine the performance of the proposed scheme against the number $F$ of fibers in each link. Figure 8 shows the blocking probability of lightpath establishment as a function of the offered load $\rho$, where $W=4$ and $F=12$. As we can see from this figure, the proposed scheme exhibits an excellent performance like the result with $F=4$ shown in Figure 7. Figure 9 shows the blocking probability of lightpath establishment as a function of the number $F$ of fibers in each link, where $W=4$ and $\rho=0.6$. As shown in this figure, the blocking probability of each scheme decreases with the increase in $F$ because multifiber links fill the role of limited-range wavelength conversion. We also observe that the proposed scheme efficiently reduces the blocking probability, regardless of the number $F$ of fibers in each link.

Next, we examine the performance of the proposed scheme against the number $W$ of wavelengths supported by each fiber. Figure 10 shows the blocking probability of lightpath establishment as a function of the offered load $\rho$, where $W=12$ and $F=4$. As we can see from this figure, SR has the high blocking probability. We also observe that the proposed scheme reduces the blocking probability more efficiently than WLCR-FF. This result is similar to the result with $W=4$ shown in Figure 7. Figure 11 shows the blocking probability of lightpath establishment as a function of the number $W$ of wavelengths supported by each fiber, where $F=4$ and $\rho=0.6$. We observe that the blocking probability of each scheme decreases with the increase in $W$ because of the large-scale effect. We also observe that the proposed scheme reduces the blocking probability more efficiently than other schemes. We conclude that the proposed scheme efficiently reduces the blocking probability of lightpath establishment regardless of the number $W$ of wavelengths supported by each fiber.

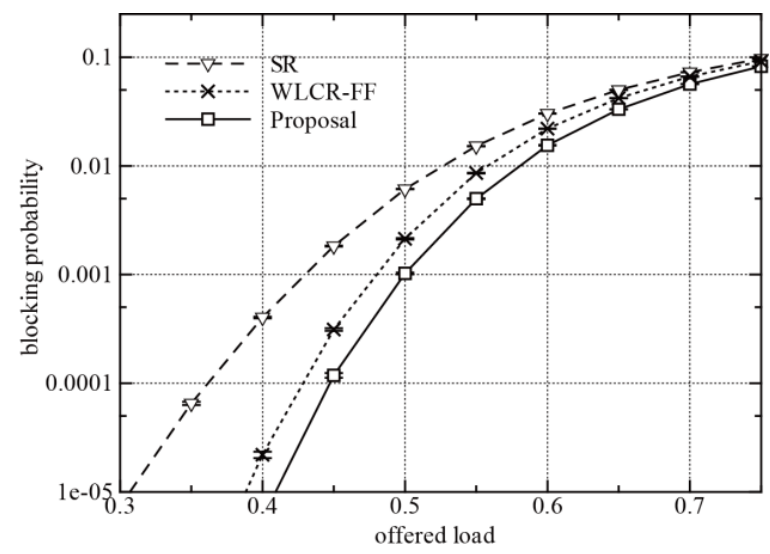

Figure 12. Blocking probability $(\mathrm{W}=4, \mathrm{~F}=4)$ (full wavelength conversion)

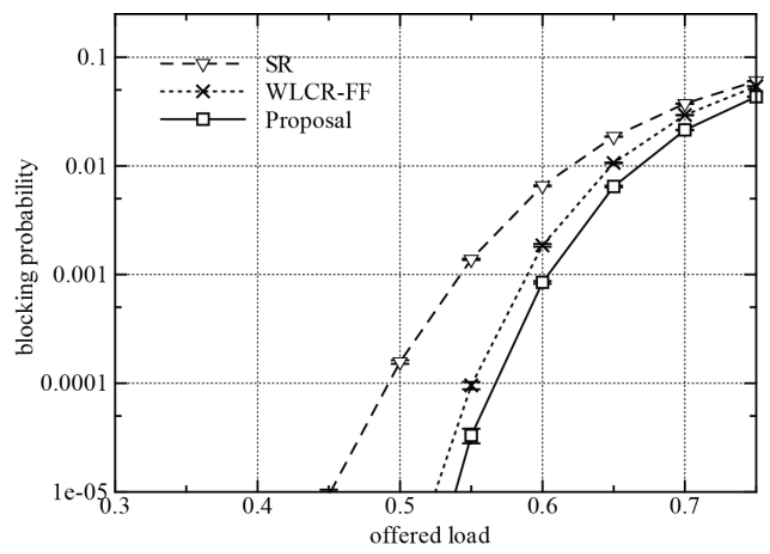

Figure 13. Blocking probability $(\mathrm{W}=4, \mathrm{~F}=12)$ (full wavelength conversion) 


\subsubsection{Full Wavelength Conversion Network}

Next, we examine the performance of the proposed scheme under full wavelength conversion environments. To do so, the number $N$ of nodes with wavelength conversion capability is set to 24 . Figure 12 shows the blocking probability of lightpath establishment as a function of the offered load $\rho$, where $W=4$ and $F=4$. We observe that the blocking probability of SR is high and the blocking probability of WLCR-FF is medium, similar to the result in sparse wavelength conversion. We also observe that the proposed scheme efficiently reduces the blocking probability.

We examine the impact of the number $F$ of fibers in each link. Figure 13 shows the blocking probability of lightpath establishment as a function of the offered load $\rho$, where $W=4$ and $F=12$. As shown in this figure, the proposed scheme exhibits an excellent performance even if the number of fibers increases. Figure 14 shows the blocking probability of lightpath establishment as a function of the number $F$ of fibers in each link. From this figure, we observe that the blocking probability of each scheme decreases with the increase of the number $F$ of fibers in each link, and the blocking probability of the proposed scheme is the smallest.

We also examine the impact of the number $W$ of wavelength supported by each fiber. Figure 15 shows the blocking probability of lightpath establishment as a function of the offered load $\rho$, where $W=12$ and $F=4$. Furthermore, Figure 16 shows the blocking probability of lightpath establishment as a function of the number $W$ of wavelengths, where $F=4$ and $\rho=0.6$. From these figures, we observe that the proposed scheme shows an excellent performance like the results in sparse wavelength conversion. Thus we conclude that the proposed scheme works efficiently, regardless of the number of nodes with wavelength conversion capability.

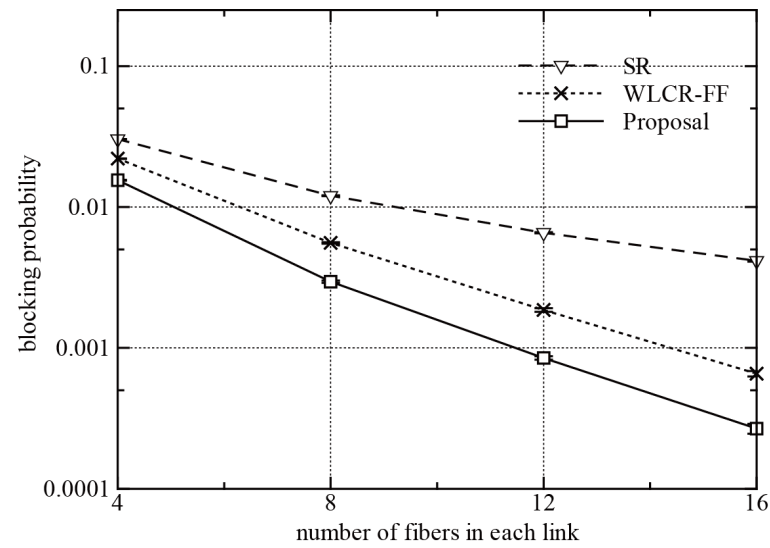

Figure 14. Blocking probability $(\mathrm{W}=4, \rho=0.6)$ (full wavelength conversion)

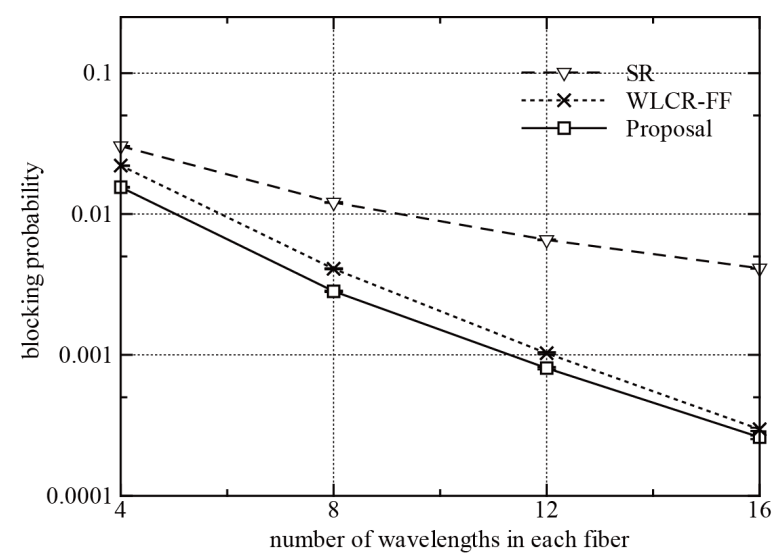

Figure 16. Blocking probability $(\mathrm{F}=4, \rho=0.6)$ (full wavelength conversion)

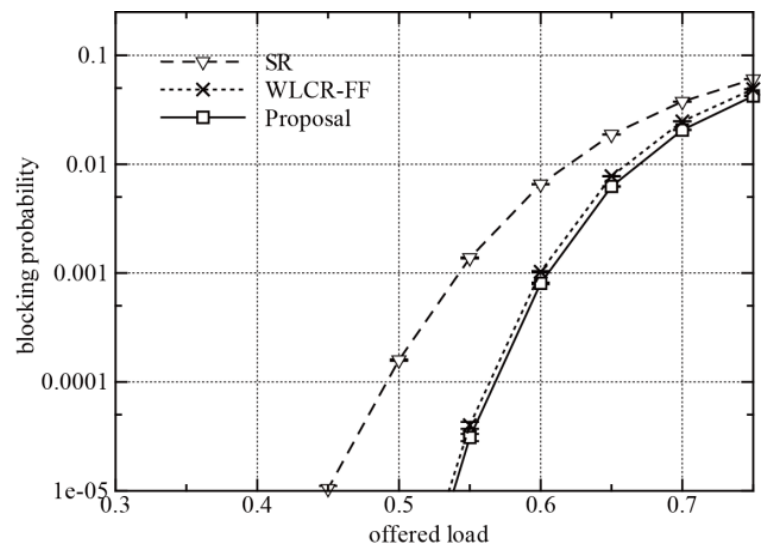

Figure 15. Blocking probability $(\mathrm{W}=12, \mathrm{~F}=4)$ (full wavelength conversion)

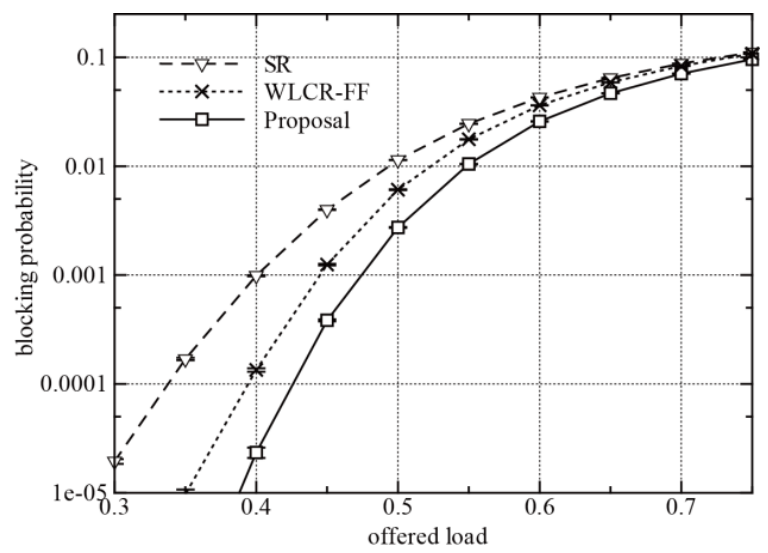

Figure 17. Blocking probability $(\mathrm{W}=4, \mathrm{~F}=4)$ (no wavelength conversion) 


\subsubsection{No Wavelength Conversion Network}

The proposed scheme is expected to efficiently work even if there are no nodes with wavelength conversion capability. To show the performance of the proposed scheme in such networks, the number $N$ of nodes with wavelength conversion capability is set to be 0 . Figure 17 shows the blocking probability of lightpath establishment as a function of the offered $\operatorname{load} \rho$, where $W=4$ and $F=4$. We observe that the blocking probability of SR is high and the blocking probability of WLCR-FF is medium. We also observe that the proposed scheme efficiently reduces the blocking probability.

Next, we examine the impact of the number $F$ of fibers in each link. Figure 18 shows the blocking probability of lightpath establishment as a function of the offered load $\rho$, where $W=4$ and $F=12$. As shown in this figure, the proposed scheme exhibits an excellent performance even if the number of fibers increases. Figure 19 shows the blocking probability of lightpath establishment as a function of the number $F$ of fibers in each link. From this figure, we observe that the blocking probability of each scheme decreases with the increase of the number $F$ of fibers in each link, and the blocking probability of the proposed scheme is the smallest.

We also examine the impact of the number $W$ of wavelengths supported by each fiber. Figure 20 shows the blocking probability of lightpath establishment as a function of the offered load $\rho$, where $W=12$ and $F=4$. Furthermore, Figure 21 shows the blocking probability of lightpath establishment as a function of the number $W$ of wavelengths, where $F=4$ and $\rho=0.6$. In these figures, the proposed scheme shows an excellent performance like the results in sparse wavelength conversion.

These results are similar to the results in sparse and full wavelength conversion. Thus we conclude that the proposed scheme work efficiently even if there are no nodes with wavelength conversion capability.

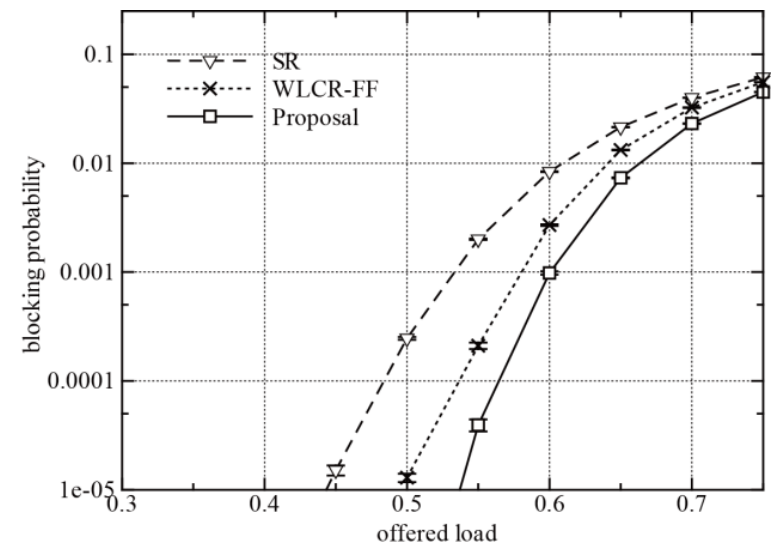

Figure 18. Blocking probability $(\mathrm{W}=4, \mathrm{~F}=12)$ (no wavelength conversion)

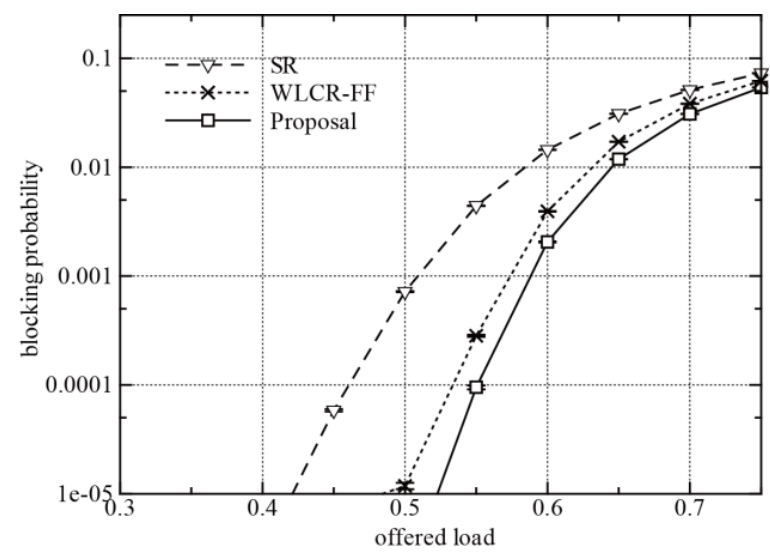

Figure 20. Blocking probability $(\mathrm{W}=12, \mathrm{~F}=4)$ (no wavelength conversion)

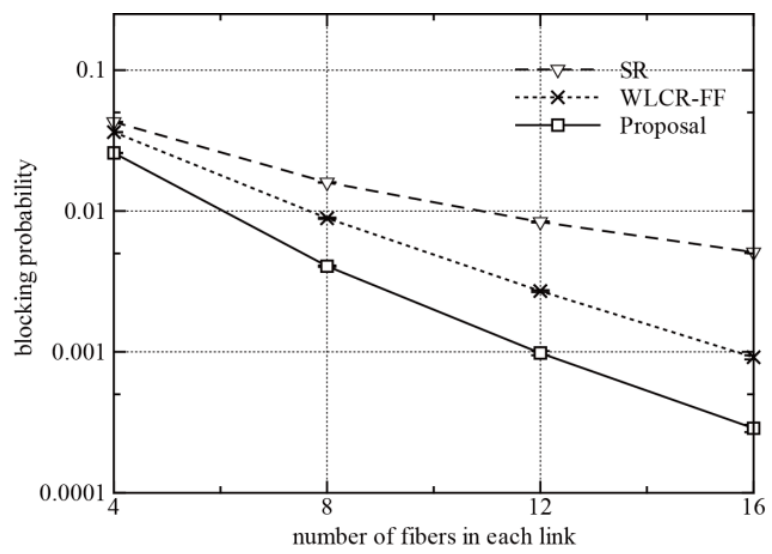

Figure 19. Blocking probability $(\mathrm{W}=4, \rho=0.6)$ (no wavelength conversion)

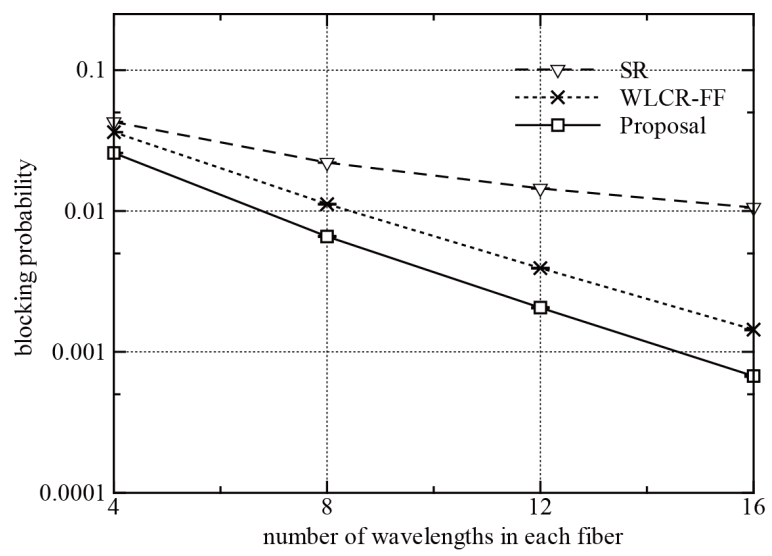

Figure 21. Blocking probability $(F=4, \rho=0.6)$ (no wavelength conversion) 


\section{Conclusion}

This paper proposed a dynamic RWA scheme for multifiber WDM networks with wavelength conversion capability. In the proposed scheme, a route and wavelengths are selected for each lightpath based on wavelength availability in links and location of nodes with wavelength conversion capability. Through simulation experiments, we observed that the proposed scheme efficiently reduces blocking probability in sparse wavelength conversion networks. Furthermore, we demonstrated the robustness of the superior performance of the proposed scheme against system parameter values such as the number of wavelengths supported by each fiber and the number of fibers in each link. We also showed that the proposed scheme works well in networks with full wavelength conversion capability and networks without wavelength conversion capability. As the future work, we will consider the effect of physical layer impairment which reduces the performance of the optical networks and propose RWA schemes to resolve the physical layer impairment problem.

\section{Acknowledgements}

This research was partially supported by Grant-in Aid for Young Scientists (B) of the Japan Society for the Promotion of Science under Grant No.23700085.

\section{References}

Baroni, S., Bayvel, P., Gibbens, R. J., \& Korotky, S. K. (1999). Analysis and design of resilient multifiber wavelength-routed optical transport networks. IEEE/OSA Journal of Lightwave Technology, 17(5), 743-758.

Chu, X., Li, B., \& Zhang, Z. (2003). A dynamic RWA algorithm in a wavelength-routed all-optical network with wavelength converters. in Proc. IEEE INFOCOM, San Francisco, CA,1795-1804.

Coiro, A., Listanti, M., \& Valenti, A. (2011a). Dynamic power-aware routing and wavelength assignment for green WDM optical networks. in Proc. IEEE International Conference on Communications (ICC 2011), Kyoto, Japan, 1-6. http://dx.doi.org/10.1109/icc.2011.5962431

Coiro, A., Listanti, M., Valenti, A., \& Matera, F. (2011b). Power-aware routing and wavelength assignment in multi-fiber optical networks. IEEE/OSA Journal of Optical Communications and Networking, 3(11), 816-829. http://dx.doi.org/10.1364/JOCN.3.000816

Dewiani, Hirata, K., Higami, Y., \& Kobayashi, S. (2011). Dynamic routing and wavelength assignment scheme using signaling of backward reservation in multifiber WDM networks. in Proc. the 2nd IEEE International Conference on ICT Convergence (ICTC2011), Seoul, Korea, 447-452.

Karasan, E., \& Banerjee, S. (1998). Performance of WDM transport networks. IEEE Journal of Selected Areas in Communications, 16(7), 1081-1096.

Kim, J. S., Lee, D. C., \& Sridhar, H. (2006). Route-metric-based dynamic routing and wavelength assignment for multifiber WDM networks. IEEE Journal of Selected Areas in Communications, 24(12), 56-68. http://dx.doi.org/10.1109/JSAC.2006.258223

Li, J., \& Somani, A. K. (2000). A new analytical model for multifiber WDM networks. IEEE Journal of Selected Areas in Communications, 18(10), 2138-2145. http://dx.doi.org/10.1109/49.887933

Liu, K. (2009). Routing and wavelength assignment algorithm in multi-fiber WDM optical networks. in Proc. the Symposium on Photonics and Optoelectronics (SOPO 2009), Wuhan, China, 1-4.

Mokhatar, A., \& Azizoglu, M. (1998). Adaptive wavelength routing in all-optical networks. IEEE/ACM Transactions on Networking, 6(2), 197-206. http://dx.doi.org/10.1109/90.664268

Nomikos, C., Pagourtzas, A., Potika, K., \& Zachos, S. (2006). Routing and wavelength assignment in multifiber WDM networks with nonuniform fiber cost. Computer Networks, 50(1), 1-14. http://dx.doi.org/10.1016/j.comnet.2004.11.028

Rahbar, A. G. P. (2010). Dynamic impairment-aware RWA in multifiber wavelength-routed all-optical networks supporting class-based traffic. IEEE/OSA Journal of Optical Communications and Networking, 2(11), 915-927. http://dx.doi.org/10.1364/JOCN.2.000915

Ramamurthy, B., \& Mukherjee, B. (1998). Wavelength conversionin WDM networking. IEEE Journal of Selected Areas in Communications, 16(7), 1061-1073.

Subramaniam, S., Azizoglu, M., \& Somani, A. K. (1996). All-optical networks with sparse wavelength conversion. IEEE/ACM Transactions on Networking, 4(4), 544-557. http://dx.doi.org/10.1109/90.532864

Xu, S., Li, L., \& Wang, S. (2000). Dynamic routing and assignment of wavelength algorithms in multifiber 
wavelength division multiplexing networks. IEEE Journal on Selected Areas in Communications, 18(10), 2130-2137. http://dx.doi.org/10.1109/49.887932

Yuan, X., Melham, R., Gupta, R., Mei, Y., \& Qiao, C. (1999). Distributed control protocols for wavelength reservation and their performance evaluation. Photonic Network Communications, 1(3), $207-218$. http://dx.doi.org/10.1023/A:1010059612474

Zang, H., Jue, J., \& Mukherejee, B. (2000). A review of routing and wavelength assignment approaches for wavelength-routed optical WDM networks. Optical Networks Magazine, 1(1), 47-60.

Zang, H., Jue, J., Sahasrabuddhe, L., Ramamurthy, R., \& Mukherjee, B. (2001). Dynamic lightpath establishment in wavelength-routed WDM networks. IEEE Communications Magazine, 39(9), 100-108. http://dx.doi.org/10.1109/35.948897 